\title{
mRCC: hohe Ansprechrate unter Sunitinib als Therapiekriterium
}

- In einer Phase-III-Zulassungsstudie zur Erstlinientherapie des metastasierten Nierenzellkarzinoms (mRCC) wurde mit Sunitinib (Sutent ${ }^{\circledast}$ ) eine objektive Ansprechrate von $47 \%$ erreicht. Bei anderen Substanzen beträgt diese etwa $30 \%$ [u.a. Sternberg $\mathrm{CN}$ et al. J Clin Oncol 2010]. Dass dies auch klinisch höchst relevant ist, unterstrich Prof. Kurt Miller, Berlin, im Rahmen des 8. AIO-Herbstkongresses. „Für viele Patienten ist es sehr motivierend, eine Verkleinerung des Tumors zu sehen - also zu sehen, dass ein Medikament das tut, was es tun soll. Zudem können durch Metastasen hervorgerufene Beschwerden gelindert werden." Aktuelle Daten weisen darauf hin, dass beim mRCC zwischen dem Ansprechen und dem Gesamtüberleben ein Zusammenhang bestehen könnte [Krajewski K et al. European Urology 2011].„Und eine Verlängerung des Überlebens ist letzt- lich das wichtigste Ziel in der heutigen mRCCTherapie", so Miller. Sunitinib sei von der deutschen interdisziplinären Konsensuskonferenz mRCC wegen der hohen Ansprechraten und der günstigen Überlebensdaten als Erstlinientherapie der Wahl bei mRCC-Patienten mit guter und mittlerer Prognose empfohlen worden., Vor der Zulassung von Sunitinib haben wir das metastasierte Nierenzellkarzinom ausschließlich mit Immuntherapeutika behandelt. Heute ist die zielgerichtete mRCC-Therapie mit Sunitinib als ein Standard in der Erstlinie nicht mehr wegzudenken", erklärte Miller. Er hob die hohe Ansprechrate beim mRCC von $47 \%$ unter Sunitinib gegenüber $12 \%$ bei Interferon-alpha ( $p$ $<0,001$ ) hervor sowie das Gesamtüberleben von 26,4 Monaten unter Sunitinib vs. 21,8 Monaten unter IFN-alpha $(p=0,051)$ [Motzer R et al. J Clin Oncol 2009].
Neben der Bedeutung des Therapieansprechens für den unmittelbaren Behandlungsverlauf wie höhere Compliance, konstruktiver Umgang mit möglichen Nebenwirkungen und Linderung von Metastasenbeschwerden lassen erste Daten einen Zusammenhang zwischen Ansprechen und Gesamtüberleben in der zielgerichteten $\mathrm{mRCC}$-Therapie vermuten: In einer US-amerikanischen Studie wurden mRCC-Patienten untersucht, die in der Erstlinie mit zielgerichteten Substanzen behandelt wurden. Es zeigte sich ein signifikant verlängertes Gesamtüberleben (OS) bei jenen Studienteilnehmern, deren Tumor sich um mindestens $10 \%$ in der Bildgebung verkleinert hatte (OS von 32,5 vs. 15,8 Monaten; $\mathrm{p}=0,002$ ) [Krajewski K et al. European Urology 2011].

Nach Informationen von Pfizer, Berlin

\section{Dreifache Pflanzenkraft hilft bei Harnwegsinfekten}

_ Jede vierte Patientin erleidet innerhalb von drei Monaten nach einer Harnwegsinfektion ein Rezidiv. Dass die Rückkehr von Brennen beim Wasserlassen und Harndrang kein unabwendbares Schicksal ist, belegen Studiendaten zur unterstützenden Therapie mit Canephron ${ }^{\circledR} \mathrm{N}$ zusätzlich zur Antibiotikatherapie bei Patienten mit einer akuten Infektion der unteren Harnwege [lvanov DD et al. 2004]. Alle 80 Patienten dieser Untersuchung erhielten aufgrund eines Harnwegsinfektes eine Standardbehandlung mit Antibiotika für drei bis 14 Tage. Davon nahmen 34 Patienten zusätzlich zur Akutbehandlung mit Antibiotika und über weitere drei Monate noch das Phytotherapeutikum Canephron ${ }^{\circledR} \mathrm{N}$ ein. Von ihnen erlitten im Beobachtungszeitraum von drei Monaten nur 5,9\% ein Rezidiv. In der Kontrollgruppe, die nur die Standardbehandlung mit Antibiotika erhielt, erlitten dagegen $21,7 \%$ erneut einen Harnwegsinfekt. Die zusätzliche Therapie mit Canephron ${ }^{\oplus} \mathrm{N}$ reduzierte die Rezidivrate damit um $73 \%$.

Canephron $\mathrm{N}$ zeichnet sich durch ein breites pharmakologisches Wirkspektrum aus. Die Kombination aus den Heilpflanzen Tausendgüldenkraut, Liebstöckel und Rosmarin kann sowohl zur Therapie akuter als auch rezidivierender Beschwerden eingesetzt werden. Die pflanzlichen Inhaltsstoffe wirken hierbei antiphlogistisch, diuretisch, spasmolytisch und antibakteriell. Infektbedingtes Brennen und Schmerzen beim Wasserlassen werden effektiv durch die antiphlogistische Wirkung bekämpft, Pathogene werden ausgespült und durch die antibakterielle Wirkung an der weiteren Vermehrung gehindert. Zudem entspannt Canephron ${ }^{\circledR} \mathrm{N}$ die glatte Blasenmuskulatur, sodass Druckgefühl und Krämpfe nachlassen.
Aufgrund seiner sehr guten Verträglichkeit kann das Phytotherapeutikum auch über mehrere Monate eingenommen werden, was dazu beiträgt, Rezidive zu verhindern. Dies steigert nicht nur die Lebensqualität der Betroffenen, sondern hilft auch der Entwicklung von Symptomen einer Reizblase vorzubeugen.

Nach Informationen von

Bionorica, Neumarkt

\section{Männerinteresse an Gesundheitsthemen steigt}

In Gesundheitsfragen haben Männer immer noch Nachholbedarf. Deshalb weitet die Online-Plattform www.männer-gerecht.de ihr Informationsangebot aus und bietet neue Inhalte zu Männergesundheit, Partnerschaft, Potenz und Fruchtbarkeit. Vor allem dem Testosteron sollte "Mann" mehr Beachtung schenken. Denn nach dem 40. Lebensjahr beginnen die „Aging Male"-Symptome eine besondere Rolle zu spielen, die unter anderem infolge abnehmender Testosteronspiegel auftreten: nachlassende Lust, Erektionsstörungen, abnehmende Muskelmasse oder eine verringerte Leistungsfähigkeit. Aus diesen Veränderungen können sich unterschiedliche Erkrankungen entwickeln, die Alltag und Beruf der Betroffenen beeinträchtigen.

Auf der Online-Plattform finden Männer nun auch Antworten auf Fragen wie „Sind Erektionsstörungen immer ein Fall für Potenzmittel?", "Was tun, wenn der Bauchumfang steigt?" oder "Wie wirkt sich Rauchen auf Potenz und Fruchtbarkeit aus?".

Nach Informationen von Ferring, Kiel 